Geometry $\&$ Topology

Volume 8 (2004) 511-538

Published: 13 March 2004

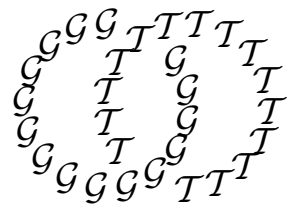

\title{
Parity of the spin structure defined by a quadratic differential
}

\author{
ERWAN LANNEAU \\ Institut de mathématiques de Luminy \\ Case 907, 163 Avenue de Luminy \\ F-13288 Marseille Cedex 9, France \\ Email: lanneau@iml.univ-mrs.fr
}

\begin{abstract}
According to the work of Kontsevich-Zorich, the invariant that classifies nonhyperelliptic connected components of the moduli spaces of Abelian differentials with prescribed singularities, is the parity of the spin structure.

We show that for the moduli space of quadratic differentials, the spin structure is constant on every stratum where it is defined. In particular this disproves the conjecture that it classifies the non-hyperelliptic connected components of the strata of quadratic differentials with prescribed singularities. An explicit formula for the parity of the spin structure is given.
\end{abstract}

AMS Classification numbers Primary: 32G15, 30F30, 30F60

Secondary: 58E12, 57R15

Keywords: Quadratic differentials, Teichmüller geodesic flow, moduli space, measured foliations, spin structure

Proposed: Benson Farb

Seconded: Jean-Pierre Otal, Shigeyuki Morita
Received: 29 July 2003

Revised: 12 March 2004 


\section{Introduction}

Quadratic differentials and moduli spaces of quadratic differentials are natural objects in Teichmüller theory. In particular, they are related to so called interval exchange transformations and billiards in rational polygons. The moduli spaces of Abelian differentials (denoted $\mathcal{H}_{g}$ ) and the moduli spaces of quadratic differentials (denoted $\mathcal{Q}_{g}$ ) are naturally stratified by the genus of surfaces and the type of the singularities of the differentials. Due to works of Veech [22] and Rauzy [20], the topology of the strata of these spaces is related to the dynamic of these intervals exchange transformations.

A fundamental result independently proved by Masur and by Veech (1982) asserts that the Teichmüller geodesic flow acts ergodically on each connected component of any stratum of the moduli spaces $\mathcal{H}_{g}$ and $\mathcal{Q}_{g}$. Recently, Kontsevich and Zorich have described the set of components for a particular type of strata: those of moduli space of Abelian differential $\mathcal{H}_{g}$ (see [12]). In order to obtain their classification, they use two invariants: the hyperellipticity and the spin structure. In a previous paper [13], we give three families of non-connected strata of $\mathcal{Q}_{g}$, using the hyperelliptic invariant. Moreover, we show that there are no other hyperelliptic components of the strata of $\mathcal{Q}_{g}$. See also [14 for a complete description of the set of components of the strata of the moduli space of quadratic differentials $\mathcal{Q}_{g}$.

In this paper we are interested in the calculation of the second invariant on the elements of $\mathcal{Q}_{g}$. Here we prove that it is constant on every stratum of $\mathcal{Q}_{g}$ where it is defined. Thus the parity of the spin structure cannot distinguish two differentials with the same singularity pattern. It is therefore a negative answer to a question of Kontsevich and Zorich on the classification of the nonhyperelliptic connected component by this spin structure. In addition we give an explicit formula for its calculation knowing the type of the singularities. In the Appendix we recall the relationship between rational billiards and quadratic differentials and then, using our formula, we give some applications in terms of billiards.

\subsection{Background}

On a Riemann surface $M_{g}^{2}$ of genus $g$, a meromorphic quadratic differential is locally defined by the form $\psi=f(z)(d z)^{2}$ where $z$ is a local coordinate. In this paper we consider quadratic differentials having only simple poles, if any: that is the functions $f$ are meromorphic with only simple poles, if any. 
Here we will use the geometric point of view of quadratic differentials: a flat structure with cone type singularities. Such surfaces are those which possesses locally the geometry of a standard cone. We can define it by a flat Riemannian metric with specific isolated singularities. The standard cone possesses a unique invariant: it is the angle at the vertex. Here we consider only half-translation flat surfaces: parallel transport of a tangent vector along any closed path either brings the vector $\vec{v}$ back to itself or brings it to the centrally-symmetric vector $-\vec{v}$. This implies that the cone angle at any singularity of the metric is an integer multiple of $\pi$.

One can see that these Euclidian structures are induced by quadratic differentials by the following way. Let $\psi$ be a meromorphic quadratic differential on a Riemann surface $M_{g}^{2}$. Then it is possible to choose a canonical atlas on $M \backslash\{$ singularities $\}$ such that $\psi=d z^{2}$ in any coordinate chart. As $d z^{2}=d w^{2}$ implies $z= \pm w+$ const, we see that the charts of the atlas are identified either by a translation or by a translation composed with a central symmetry. Thus a meromorphic quadratic differential $\psi$ induces a half-translation flat structure on $M \backslash$ singularities . On a small chart which contains a singularity, coordinate $z$ can be chosen in such way that $\psi=z^{k} d z^{2}$, where $k$ is the order of the singularity ( $k=0$ corresponds to a regular point, $k=-1$ corresponds to a pole and $k>0$ corresponds to a true zero). It is easy to check that in a neighborhood of a singularity of $\psi$, the metric has a cone type singularity with the cone angle $(k+2) \pi$.

Conversely, a half-translation structure on a Riemann surface $M$ and a choice of a distinguished "vertical" direction defines a complex structure and a meromorphic quadratic differential $\psi$ on $M$. In many cases it is very convenient to present a quadratic differential with some specific properties by an appropriate flat surface. Consider, for example, a polygon in the complex plane $\mathbb{C}$ with the following property of the boundary: the sides of the polygon are distributed into pairs, where the sides in each pair are parallel and have equal length. Identifying the corresponding sides of the boundary by translations and central symmetries we obtain a Riemann surface with a natural half-translation flat structure. The quadratic differential $d z^{2}$ on $\mathbb{C}$ gives a quadratic differential on this surface with punctures. The punctures correspond to vertices of the polygon; they produce the cone type singularities on the surface. It is easy to see that the complex structure, and the quadratic differential extends to these points, and that a singular point of the flat metric with a cone angle $(k+2) \pi$ produces a singularity of order $k$ (a pole if $k=-1$ ) of the quadratic differential. (See also Figure 1 which illustrates this construction.) 
Moduli Spaces According to these definitions, one can define the moduli space $\mathcal{H}_{g}$ of Abelian differentials as the moduli space of pairs $(M, \omega)$ where $\omega$ is a holomorphic 1 -form defined on a Riemann surface $M$ of genus $g$. Here the term moduli spaces means that the points $\left(M_{1}, \omega_{1}\right),\left(M_{2}, \omega_{2}\right)$ are identified if and only if there exists an isomorphism $f: M_{1} \rightarrow M_{2}$ (with respect to the complex structure) affine in the canonical chart determined by $\omega_{i}$. In an equivalent way we can ask that $f^{*} \omega_{2}=\omega_{1}$.

We can also define the moduli space $\mathcal{Q}_{g}$ of quadratic differentials as the moduli space of pairs $(M, \psi)$ where $\psi$ is a meromorphic quadratic differential which is not the global square of a 1 -form defined on $M$.

Recall that we denote by $\left(k_{1}, \ldots, k_{n}\right)$ the orders of singularities of $\psi$. A consequence of the Gauss-Bonet formula is that $\sum k_{i}=4 g-4$.

Stratification The moduli space $\mathcal{Q}_{g}$ is naturally stratified by the types of singularities of the forms. We denote by $\mathcal{Q}\left(k_{1}, \ldots, k_{n}\right) \subset \mathcal{Q}_{g}$ the stratum of quadratic differentials $\left[M_{g}^{2}, \psi\right] \in \mathcal{Q}_{g}$ which are not the squares of Abelian differentials, and which have the singularity pattern $\left(k_{1}, \ldots, k_{n}\right)$, where $k_{i} \in$ $\{-1,0,1,2, \ldots\}$. We use the exponential notation $k_{i}^{m}$ for $k_{i}, k_{i}, k_{i}, \ldots, k_{i}$ repeated $m$ times. For example, $\mathcal{Q}\left(1^{4}, 8,2,3^{2}\right)$ stands for $\mathcal{Q}(1,1,1,1,8,2,3,3)$.

We can also consider the moduli space of Abelian differentials or quadratic differentials which are the global square of Abelian differentials. We denote these spaces by $\mathcal{H}_{g}$. And, if $\vec{k}$ is a vector in $\mathbb{N}^{n}$ with $\sum k_{i}=2 g-2$, we denote by $\mathcal{H}\left(k_{1}, \ldots, k_{n}\right)$ the corresponding stratum.

Teichmüller Geodesic Flow The group $\operatorname{SL}(2, \mathbb{R})$ acts on these spaces $\mathcal{Q}_{g}$ and $\mathcal{H}_{g}$ in the following way. For a matrix $A \in \mathrm{SL}(2, \mathbb{R})$ and a point $[M, \psi]$ we define $A[M ; \psi]$ by the point $[M ; A \psi]$. Notation $A \psi$ means $A$ acts linearly in the canonical charts determined by $\psi$. By definition, this action preserves each stratum. The following one-parameter subgroup are of special interest:

$$
g_{t}=\left(\begin{array}{cc}
e^{t / 2} & 0 \\
0 & e^{-t / 2}
\end{array}\right)
$$

We call it the Teichmüller geodesic flow.

In classical works, Masur and Veech discovered that the geodesic flow on the moduli space of quadratic differentials $\mathcal{H}_{g} \sqcup \mathcal{Q}_{g}$ is related to the theory of the so-called interval exchange transformation. The phase space of the Teichmüller geodesic flow can be seen as the cotangent bundle to the Teichmüller space, and 
it can be interpreted as the moduli space of pairs consisting of a Riemann surface endowed with a holomorphic quadratic differential. It is well known that the flow preserves the natural stratification and that each stratum carries a complex algebraic orbifold structure. Moreover, Masur and Smillie have proved that all of these strata, except four particular cases in low genera, are non-empty.

The study of the topology of the strata comes from a fundamental Theorem, independently proved by Masur and Veech.

Theorem (Masur, Veech, 1982) The Teichmüller geodesic flow acts ergodically on every connected component of any stratum with respect to a finite equivalent Lebesgue measure.

Kontsevich and Zorich have recently described the set of connected components for a particular type of strata: one of the moduli space of Abelian differentials $\mathcal{H}_{g}$ [12. In two papers [13, 14, we have obtained the "complementary" case, namely the description of components of the moduli space of quadratic differentials $\mathcal{Q}_{g}$.

In their classification, Kontsevich and Zorich use two invariants to obtain a complete description of the components: the hyperellipticity and the parity of the spin structure. These invariants allow them to show that each stratum of the moduli space $\mathcal{H}_{g}$ possesses at most 3 components.

In this paper, we are interested in the computation of the second invariant in $\mathcal{Q}_{g}$, that is the parity of the spin-structure of an arbitrary quadratic differential. Our main result is that this invariant is constant on every stratum of $\mathcal{Q}_{g}$ where it is defined. It is therefore a negative result in contrast to that of KontsevichZorich.

\subsection{Formulation of the statement}

Given an Abelian differential $\omega$ with even zeroes, one can associate the following equation in the Picard group $\operatorname{Pic}\left(M_{g}^{2}\right)$

$$
2 \cdot K=D(\omega)=\sum_{i=1}^{n} 2 \cdot k_{i} P_{i}
$$

We define the spin structure determined by $\omega$ by $\sum_{i=1}^{n} k_{i} P_{i}$. The parity of the spin structure is defined as $\Phi(\omega)=\operatorname{dim}\left|\sum_{i=1}^{n} k_{i} P_{i}\right| \bmod 2$. Following works of M Atiyah and D Mumford [2, 19], it can be shown that this integer is invariant 
under continuous deformation. Moreover the spin-structure is the basic invariant which allows to classify all non-hyperelliptic connected components of any stratum of $\mathcal{H}_{g}$.

In section 2, we consider a canonical local mapping (induced by the standard orientating double covering)

$$
\mathcal{Q}\left(k_{1}, \ldots, k_{n}\right) \longrightarrow \mathcal{H}\left(\tilde{k}_{1}, \ldots, \tilde{k}_{r}\right) .
$$

Using this mapping, we associate to each pair $\left[M_{g}^{2}, \psi\right]$ an integer 0 or 1 which we defined as the parity of the spin structure determined by $\psi$. This integer is invariant under continuous deformation of the point $\left[M_{g}^{2}, \psi\right]$ in the given stratum.

In section 4 we show that for quadratic differentials the spin-structure is constant on every stratum. Moreover we give an explicit formula to determine it knowing the singularity pattern $\left(k_{1}, \ldots, k_{n}\right)$ of the stratum. This gives a negative response to a question of Kontsevich and Zorich that it may distinguish the non-hyperelliptic connected components of some strata $\mathcal{Q}\left(k_{1}, \ldots, k_{n}\right)$. More precisely, we will show

Theorem 1.1 Let $\psi$ be a quadratic differential on a Riemann surface such that $\psi$ does not possess zeroes of order $k$ with $k=2 \bmod 4$. This condition assures that the spin structure of $\psi$ is well defined. Then the parity of the spin structure of $\psi$ is independent of the choice of $\psi$ in a stratum $\mathcal{Q}\left(k_{1}, \ldots, k_{l}\right)$.

In addition, we end section 4 by the following description Theorem in terms of $k_{i}$.

Theorem 1.2 Let $\psi$ be a meromorphic quadratic differential on a Riemann surface $M$ with singularity pattern $\mathcal{Q}\left(k_{1}, \ldots, k_{l}\right)$. We also require that $k_{i} \neq 2$ mod 4. Let $n_{+1}$ be the number of zeros of $\psi$ of degrees $k_{i}=1 \bmod 4$, let $n_{-1}$ be the number of zeros of $\psi$ of degrees $k_{j}=3 \bmod 4$, and suppose that the degrees of all the remaining zeros satisfy $k_{r}=0 \bmod 4$. Then the parity of the spin structure defined by $\psi$ is given by

$$
\Phi(\psi)=\left[\frac{\left|n_{+1}-n_{-1}\right|}{4}\right] \bmod 2
$$

where square brackets denote the integer part. 


\subsection{Deformation of flat surfaces}

Here, we explicitly present an example to illustrate that the problem of classification of component is quite difficult. For this, let us consider the domain given by Figure 1 in $\mathbb{C}$ endowed with its complex structure induced by the form $d z$. We identify the opposite sides by a translation (with respect to the numbered of sides). We get a Riemann surface of genus $g=3$ with an Abelian differential induced from the form $d z$. It is easy to see that, according to previous notations, we obtain a point in the stratum $\mathcal{H}(2,2)$.

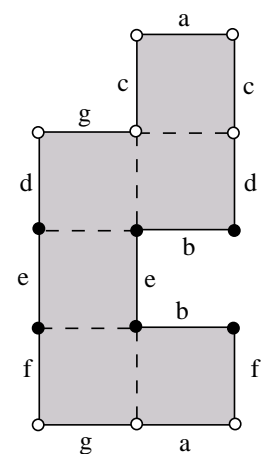

Figure 1: A point in the stratum $\mathcal{H}(2,2)$

Now we can make some surgery on this polygon: we glue a small rectangle on the boundary of the polygon and identified the vertical boundary of the small additional rectangle with appropriate translations (see Figure 2 for details). As above, we make identifications of opposite sides according the numbered of sides. We get a Riemann surface. It is not difficult to see that it has genus $g=4$. The two Abelian differentials induced from the form $d z$ in the complex plane have one zero of order 2 and one zero of order 4 (given respectively by black and white bullets). Thus, with our above notation, we obtain two points in the stratum $\mathcal{H}(2,4)$. Let denote them respectively by $\left(M, \omega_{1}\right)$ and $\left(M, \omega_{2}\right)$.

It is difficult to see directly if these two points belong in the same connected component of the stratum $\mathcal{H}(2,4)$ or not. In this case, using the parity of the spin structure, we can check that $\omega_{1}$ and $\omega_{2}$ are not in the same connected component (one can see this by the fact that there exists an additional handle and then we can apply Lemma 11 of [12]).

However, we can perform an analogous surgery to the first one (see Figure 31)

In the same way, we obtain two other points in the stratum $\mathcal{H}(2,4)$. Let us denote them by $\left[M, \omega_{3}\right]$ and $\left[M, \omega_{4}\right]$. Using the parity of the spin structure, 


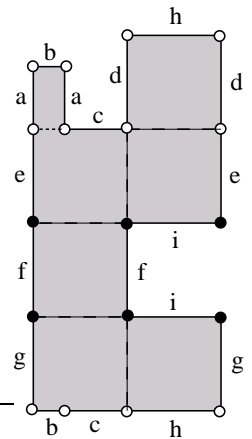

$\left(M, \omega_{1}\right)$

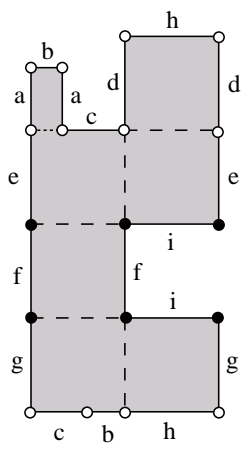

$\left(M, \omega_{2}\right)$

Figure 2: Two points $\left(M, \omega_{1}\right)$ and $\left(M, \omega_{2}\right)$ in the stratum $\mathcal{H}(2,4)$

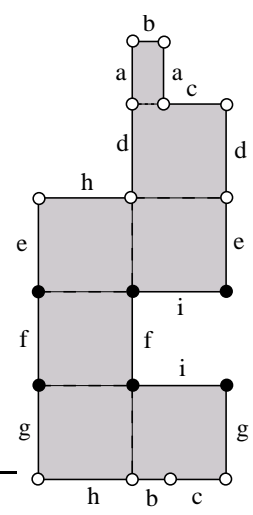

$\left(M, \omega_{3}\right)$

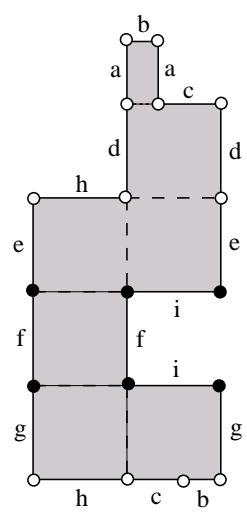

$\left(M, \omega_{4}\right)$

Figure 3: Two points $\left(M, \omega_{3}\right)$ and $\left(M, \omega_{4}\right)$ in the stratum $\mathcal{H}(2,4)$

we can deduce that these two points are in the same component. In fact, using notations of 12, we have

$$
\left[M, \omega_{1}\right],\left[M, \omega_{3}\right],\left[M, \omega_{4}\right] \in \mathcal{H}^{\text {odd }}(2,4) \quad \text { and } \quad\left[M, \omega_{2}\right] \in \mathcal{H}^{\text {even }}(2,4)
$$

However, it seems very difficult to prove this fact directly.

The paper has the following structure: we present some general facts about quadratic differentials in section 2. In section 4 we announce and prove the main Theorem. In section 4.3 we give the formula to compute the parity of the spin structure. In the Appendix we recall some classical constructions related to rational polygonal billiards and we use our formula to obtain applications in this context. 


\subsection{Acknowledgements}

I would like to thank Anton Zorich for discussion on this subject and remarks concerning this paper. I thank the Institut de Mathématiques de Luminy and the Max-Planck-Institut für Mathematik at Bonn for excellent welcome and working conditions.

\section{Mapping of the moduli spaces induced by a rami- fied covering of a fixed combinatorial type}

In this section we present some general information concerning the moduli spaces of quadratic differentials. The proofs and the details can be found in papers [15, 22, 16, 23, 11, 12, 3].

Theorem (Masur and Smillie) Consider a vector $\left(k_{1}, \ldots, k_{n}\right)$ with all $k_{i} \in$ $\mathbb{N} \cup\{-1\}$. Suppose that $\sum k_{i}=0 \bmod 4$ and $\sum k_{i} \geq-4$. The corresponding stratum $\mathcal{Q}\left(k_{1}, \ldots, k_{n}\right)$ is non-empty with the following four exceptions:

$$
\mathcal{Q}(\emptyset), \mathcal{Q}(1,-1) \text { (in genus } g=1 \text { ) and } \mathcal{Q}(4), \mathcal{Q}(1,3) \text { (in genus } g=2 \text { ) }
$$

Theorem (Masur, Veech) Any stratum $Q\left(k_{1}, \ldots, k_{n}\right)$ is a complex orbifold of dimension

$$
\operatorname{dim}_{\mathbb{C}} \mathcal{Q}\left(k_{1}, \ldots, k_{n}\right)=2 g+n-2 .
$$

Proposition (Kontsevich) Any stratum $\mathcal{Q}\left(k_{1}, \ldots, k_{n}\right)$ with $\sum k_{i}=-4$ is connected.

Here we recall a classical construction of the "orientating map" of a quadratic differential. We refer to 3 for a general reference.

Construction (Canonical double covering) Let $M_{g}^{2}$ be a Riemann surface and let $\psi$ be a quadratic differential on it which is not a square of an Abelian differential. There exists a canonical (ramified) double covering $\pi: \tilde{M}_{\tilde{g}}^{2} \rightarrow M_{g}^{2}$ such that $\pi^{*} \psi=\tilde{\omega}^{2}$, where $\tilde{\omega}$ is an Abelian differential on $\tilde{M}_{\tilde{g}}^{2}$.

The images $P \in M_{g}^{2}$ of ramification points of the covering $\pi$ are exactly the singularities of odd degrees of $\psi$. The covering $\pi: \tilde{M}_{\tilde{g}}^{2} \rightarrow M_{g}^{2}$ is the minimal (ramified) covering such that the quadratic differential $\pi^{*} \psi$ becomes the square of an Abelian differential on $\tilde{M}_{\tilde{g}}^{2}$. 
Proof Consider an atlas $\left(U_{i}, z_{i}\right)_{i}$ on $\stackrel{\circ}{M}_{g}^{2}=M_{g}^{2} \backslash\{$ singularities of $\psi\}$ where we punctured all zeros and poles of $\psi$. We assume that all the charts $U_{i}$ are connected and simply-connected. The quadratic differential $\psi$ can be represented in this atlas by a collection of holomorphic functions $f_{i}\left(z_{i}\right)$, where $z_{i} \in U_{i}$, satisfying the relations:

$$
f_{i}\left(z_{i}\left(z_{j}\right)\right) \cdot\left(\frac{d z_{i}}{d z_{j}}\right)^{2}=f_{j}\left(z_{j}\right) \text { on } U_{i} \cap U_{j}
$$

Since we have punctured all singularities of $\psi$ any function $f_{i}\left(z_{i}\right)$ is nonzero at $U_{i}$. Consider two copies $U_{i}^{ \pm}$of every chart $U_{i}$ : one copy for every of the two branches $g_{i}^{ \pm}\left(z_{i}\right)$ of $g^{ \pm}\left(z_{i}\right):=\sqrt{f_{i}\left(z_{i}\right)}$ (of course, the assignment of " + " or "-" is not canonical). Now for every $i$ identify the part of $U_{i}^{+}$corresponding to $U_{i} \cap U_{j}$ with the part of one of $U_{j}^{ \pm}$corresponding to $U_{j} \cap U_{i}$ such that on the overlap the branches match:

$$
g_{i}^{+}\left(z_{i}\left(z_{j}\right)\right) \cdot \frac{d z_{i}}{d z_{j}}=g_{j}^{ \pm}\left(z_{j}\right) \text { on } U_{i}^{+} \cap U_{j}^{ \pm}
$$

Apply the analogous identification to every $U_{i}^{-}$. We get a Riemann surface with punctures provided with a holomorphic 1-form $\tilde{\omega}$ on it, where $\tilde{\omega}$ is presented by the collection of holomorphic functions $g_{i}^{ \pm}$in the local charts. It is an easy exercise to check that filling the punctures we get a closed Riemann surface $\tilde{M}_{\tilde{g}}^{2}$, and that $\tilde{\omega}$ extends to an Abelian differential on it. We get a canonical (possibly ramified) double covering $\pi: \tilde{M}_{\tilde{g}}^{2} \rightarrow M_{g}^{2}$ such that $\pi^{*} \psi=\tilde{\omega}^{2}$.

By construction the only points of the base $M_{g}^{2}$ where the covering might be ramified are the singularities of $\psi$. In a small neighborhood of zero of even degree $2 k$ of $\psi$ we can choose coordinates in which $\psi$ is presented as $z^{2 k}(d z)^{2}$. In this chart we get two distinct branches $\pm z^{k} d z$ of the square root. Thus the zeros of even degrees of $\psi$ and the marked points are the regular points of the covering $\pi$. However, it easy to see that the covering $\pi$ has exactly a ramification point over any zero of odd degree and over any simple pole of $\psi$.

Remark 1 We can consider this construction for any quadratic differential. Note that corresponding quadratic differential is the square of an Abelian differential if and only if $\tilde{M}_{g}$ is non-connected.

Now, consider a non-degenerate point $[M, \varphi] \in \mathcal{Q}\left(k_{1}, \ldots, k_{n}\right)$, that is a nonorbifoldic point. Deforming slightly the initial point $\left[M_{g}^{2}, \psi_{0}\right] \in \mathcal{Q}\left(k_{1}, \ldots, k_{n}\right)$ 
we can consider the canonical double ramified covering over the deformed Riemann surface of the same combinatorial type as the covering $\pi$. This new covering has exactly the same relation between the positions and types of the ramification points and the degrees and position of singularities of the deformed quadratic differential. This means that the induced quadratic differential $\pi^{*} \psi$ has the same singularity pattern. Choosing one of two branches of $\omega= \pm \sqrt{\pi^{*} \psi}$, we get a local mapping:

$$
\begin{gathered}
\mathcal{Q}\left(k_{1}, \ldots, k_{n}\right) \rightarrow \mathcal{H}\left(\tilde{k}_{1}, \ldots, \tilde{k}_{m}\right) \\
{\left[M_{g}^{2}, \psi\right] \mapsto\left(\tilde{M}_{g}^{2}, \pi^{*} \psi\right)}
\end{gathered}
$$

Remark 2 A detailed proof of this fact can be found in [13.

\section{$3 \quad$ Parity of a spin structure defined by a quadratic differential}

\subsection{Spin structure defined by an Abelian differential}

We first recall the algebraic-geometric definition of the spin structure given by an Abelian differential, see M Atiyah [2]; see also [12; see D Johnson [10] for a topological definition.

\subsubsection{Definition of a spin structure}

Let $\omega$ be a 1 -form with only even singularities. There are $2^{2 g}$ solutions of the equation $2 D=K(\omega)$ in the divisor group where $K(\omega)$ is the canonical divisor determined by $\omega$. A spin structure is the choice of $D$ in the Picard group of the surface. For an Abelian differential with only even zeros, one can write

$$
K(\omega)=2 k_{1} P_{1}+\cdots+2 k_{n} P_{n}
$$

With these notation, we declare that the spin structure defined by the form $\omega$ on the complex curve $M$ is just the divisor $D=k_{1} P_{1}+\cdots+k_{n} P_{n}$. Thus a point $(M, \omega)$ gives canonically a spin structure.

\subsubsection{Parity of a spin structure}

The dimension of the linear space $|D|$ may have quite different values for different choice of $D$. For example, in genus 1, the dimension of the spaces given 
by the three non-null solutions have non-zero dimension. We declare that the dimension modulo 2 of this linear space is the parity of the spin structure $D$ and we denote it by $\Phi(D)$. On a curve of genus $g \geq 1$, M Atiyah [2] proved that there are $2^{g-1}\left(2^{g}+1\right)$ odd spin structure and $2^{2 g}-2^{g-1}\left(2^{g}+1\right)$ even spin structure.

\subsubsection{Calculation of the parity of a spin structure}

Let $M$ be a Riemann surface with an Abelian differential $\omega$. Denote by $\stackrel{\circ}{M}$ the surface $M$ punctured at the singularities of $\omega$. Consider the flat metric on $\stackrel{\circ}{M}$ defined by $\omega$. The corresponding holonomy representation in the linear group is trivial: a parallel transport of a tangent vector along any closed loop brings the vector to itself. Thus we get a canonical trivialization of the tangent bundle $T_{*} \stackrel{\circ}{M}$ to the punctured Riemann surface $\stackrel{\circ}{M}$ : the tangent space at any point of $\stackrel{\circ}{M}$ is canonically identified with a sample Euclidean plane $\mathbb{R}^{2}$.

Let $\gamma$ be a closed smooth oriented connected curve on $M$ avoiding singularities of $\omega$. Using the trivialization of the tangent bundle to $\stackrel{\circ}{M}$ we can construct the Gauss map $\left(\mathcal{G}: \gamma \rightarrow S^{1}\right)$ as follows: we associate to a point $x \in \gamma$ the image of the normalized tangent vector to $\gamma$ at $x$ under the trivialization map $T_{1} \stackrel{\circ}{M} \rightarrow S^{1} \subset \mathbb{R}^{2}$. Here the unit tangent bundle $T_{1} \stackrel{\circ}{M} \subset T_{*} \stackrel{\circ}{M}$ to $\stackrel{\circ}{M}$ is defined in terms of the flat metric on $\stackrel{\circ}{M}$ defined by $\omega$.

The index of the closed curve $\gamma$ in the flat metric defined by $\omega$ is defined to be the degree of the Gauss map

$$
\operatorname{ind}_{\gamma}(\omega):=\operatorname{deg}\left(\mathcal{G}: \gamma \rightarrow S^{1}\right) .
$$

In other words, following the closed curve $\gamma$ in the positive direction and measuring how the tangent vector turns in the flat structure we get the angle $\operatorname{ind}_{\gamma}(\omega) \cdot 2 \pi$ as a total turn along $\gamma$. For example, given a zero of order $k \geq 0$ and a small curve $\gamma$ around it, we get for the index of $\gamma(k+1) 2 \pi$.

Remark 3 If $\triangle$ is a small disc in $M$ avoiding singularities, by definition $[\partial \triangle]=0$ in $H_{1}\left(\stackrel{\circ}{M} ; \mathbb{Z}_{2}\right)$. Thus we have

$$
\begin{aligned}
& \Omega_{\omega}(\partial \triangle)=\Omega_{\omega}(\partial \triangle+\partial \triangle)= \\
& \quad=\Omega_{\omega}(\partial \triangle)+\Omega_{\omega}(\partial \triangle)+\partial \triangle \\
& \quad=2 \cdot \Omega_{\omega}(\partial \triangle)+0=0 \bmod 2
\end{aligned}
$$

where $\circ$ stands for the standard intersection form on $H_{1}\left(M ; \mathbb{Z}_{2}\right) \bmod 2$. 
Moreover, if we have only one singularity inside $\triangle$ of order $k$ we have

$$
\Omega_{\omega}(\partial \triangle)=\operatorname{ind}_{\partial \triangle}(\omega)+1=k \bmod 2 .
$$

We obtain $k=0 \bmod 2$ thus $\omega$ must possesses only singularities of even order.

Remark 3 implies that when the Abelian differential $\omega$ has zeros of even degree only, the residue $\operatorname{ind}_{\gamma}(\omega) \bmod 2$ depends only on the homology class $[\gamma] \in$ $H_{1}\left(M ; \mathbb{Z}_{2}\right)$ of the smooth closed connected path $\gamma$.

This invariance allows to define the function $\Omega_{\omega}: H_{1}\left(M ; \mathbb{Z}_{2}\right) \rightarrow \mathbb{Z}_{2}$ : to evaluate $\Omega_{\omega}$ on a cycle $c \in H_{1}\left(M ; \mathbb{Z}_{2}\right)$ we represent $c \in H_{1}\left(M ; \mathbb{Z}_{2}\right)$ by a closed smooth oriented connected curve $\gamma$ on $M$ avoiding singularities of $\omega$, and define

$$
\Omega_{\omega}(c):=\operatorname{ind}_{\gamma}(\omega)+1 \bmod 2 .
$$

The function $\Omega_{\omega}$ is, actually, a quadratic form on $H_{1}\left(M ; \mathbb{Z}_{2}\right)$, that is

$$
\Omega_{\omega}\left(c_{1}+c_{2}\right)=\Omega_{\omega}\left(c_{1}\right)+\Omega_{\omega}\left(c_{2}\right)+c_{1} \circ c_{2} .
$$

Let $\left\{a_{1}, b_{1}, \ldots, a_{g}, b_{g}\right\}$ be a symplectic basis of $H_{1}(M, \mathbb{Z})$. We define the Arfinvariant of the quadratic form $\Omega_{\omega}$ as follows:

$$
\Phi\left(\Omega_{\omega}\right)=\sum_{i=1}^{g} \Omega_{\omega}\left(a_{i}\right) \Omega_{\omega}\left(b_{i}\right) \bmod 2
$$

According to [1], the number defined by the above formula, is independent of the choice of a symplectic basis of cycles. In addition, Johnson [10 proved that the set of quadratic forms and spin structures is one to one. Finally, Johnson has shown that the two numbers (modulo 2) defined by the parity of the spin structure and the Arf invariant of the corresponding quadratic form must coincide. Thus we have

$$
\Phi(\omega)=\Phi(K(\omega))=\operatorname{dim}|D(\omega)| \bmod 2=\Phi\left(\Omega_{\omega}\right)
$$

where $D(\omega)=k_{1} P_{1}+\cdots+k_{n} P_{n}$, and $2 k_{i}$ is the multiplicity of the zero $P_{i}$ of $\omega$.

\subsubsection{Spin structure on a deformed curve}

According the result of M Atiyah [2] and D Mumford [19, the dimension of the linear space $|D(\omega)|$ modulo 2 is invariant under continuous deformations of the Abelian differential $\omega$ inside the corresponding stratum, and hence, it is constant for every connected component of any stratum, where it is defined. 


\subsection{Spin structure on a double covering defined by a quadratic differential}

Now we want to define the parity of the spin structure for a quadratic differential. Recall that this number is well defined only for Abelian differentials with zeroes of even orders.

Let $M$ be a Riemann surface and $\psi$ a quadratic differential on $M$ which is not the square of any Abelian differential. We suppose that orders of all singularities of $\psi$ are different from 2 modulo 4 . We consider the canonical (branched) double covering $\pi: \tilde{M} \rightarrow M$ over $M$ such that $\pi^{*} \psi=\omega^{2}$ (see Construction in Section (2). Then we can check that condition on the degree of the zeroes of $\psi$ implies that $\omega$ possesses only zeros of even order (Lemma 1 in [13]). So we can apply the notion of parity of the spin structure to $\omega$. With these notation, we declare that the parity of spin structure of $\psi$ is the parity of the spin structure determined by $\omega$ :

$$
\Phi(\psi) \stackrel{\text { Def }}{=} \Phi(\omega)
$$

As $\omega$ and $-\omega$ are in the same connected component (consider the path $e^{i \pi t} \omega$ for $t \in[0,1])$, we have $\Phi(\omega)=\Phi(-\omega)$. So $\Phi(\psi)$ is well-defined.

\section{Invariance of the parity of the spin structure}

In this Section, we prove the announced result.

\subsection{Monodromy representation for the canonical double cover- ing}

Let $M$ be a Riemann surface with $\psi$ a quadratic differential, that is not the square of an Abelian differential. Let $\pi: \tilde{M} \rightarrow M$ be the canonical (ramified) double covering such that the pullback $\pi^{*} \psi$ of $\psi$ to the covering surface $\tilde{M}$ becomes the global square of an Abelian differential, $\pi^{*}(\psi)=\omega^{2}$. Removing from $\tilde{M}$ the ramification points (if there are any), and removing the singularities of $\psi$ of odd degrees (if there are any) from $M$ we obtain a regular $\mathbb{Z}_{2}$-covering

$\pi: \tilde{M} \backslash\{$ ramification points $\} \rightarrow M \backslash$ singularities of $\psi$ of odd degree $\}$

Denote $\stackrel{\circ}{M}:=M \backslash$ singularities of $\psi$ of odd degree $\}$. We get a monodromy representation $m: \pi_{1}(\stackrel{\circ}{M}) \rightarrow \mathbb{Z}_{2}$. Since $\mathbb{Z}_{2}$ is an Abelian group the monodromy 
representation quotients as $\pi_{1}(\stackrel{\circ}{M}) \rightarrow H_{1}(\stackrel{\circ}{M} ; \mathbb{Z}) \rightarrow \mathbb{Z}_{2}$, and even as $\pi_{1}(\stackrel{\circ}{M}) \rightarrow$ $H_{1}\left(\stackrel{\circ}{M} ; \mathbb{Z}_{2}\right) \rightarrow \mathbb{Z}_{2}$. We denote the monodromy homomorphism $m: H_{1}\left(\stackrel{\circ}{M} ; \mathbb{Z}_{2}\right) \rightarrow$ $\mathbb{Z}_{2}$ by the same symbol $m$.

A closed connected path $\gamma$ on $\stackrel{\circ}{M}$ lifts to a closed path on the covering surface $\tilde{M}$ if and only if the homology class $[\gamma] \in H_{1}\left(\stackrel{\circ}{M} ; \mathbb{Z}_{2}\right)$ belongs to $\operatorname{Ker}(m)$. Note, however, that if $\psi$ does have singularities of odd degree it is always possible to modify a closed connected path $\gamma$ on $\stackrel{\circ}{M}$ in such way that the modified closed connected path $\gamma^{\prime}$ stays in the same homology class $[\gamma]=$ $\left[\gamma^{\prime}\right] \in H_{1}\left(M ; \mathbb{Z}_{2}\right)$, but the monodromy along $\gamma$ and $\gamma^{\prime}$ are different, $m(\gamma) \neq$ $m\left(\gamma^{\prime}\right)$. The reason is that the homology classes of $\gamma$ and $\gamma^{\prime}$ in homology of the punctured surface $H_{1}\left(\stackrel{\circ}{M} ; \mathbb{Z}_{2}\right)$ are different. In example presented by Figure 4 the difference corresponds to a cycle turning around the puncture, and such a cycle has nontrivial monodromy.

Note, that if a cycle in $H_{1}\left(\stackrel{\circ}{M} ; \mathbb{Z}_{2}\right)$ is represented by a smooth closed connected curve $\gamma$ on $M$ which does not pass through singularities of $\psi$, the monodromy $m(\gamma)$ can be calculated geometrically. It is trivial if and only if the holonomy of the flat metric defined by $\psi$ along this path is trivial - a parallel transport of a vector along the path brings the vector to itself.

\subsection{Main result}

Now we have all necessary tools to prove the following Theorem.

Theorem 4.1 The parity of the spin structure of a quadratic differential $\psi$, that is not the square of an Abelian differential, is independent of the choice of $\psi$ in a stratum $\mathcal{Q}\left(k_{1}, \ldots, k_{l}\right)$.

Proof of the Theorem 4.1 We first present an idea of the proof. Let $M$ be a Riemann surface with a quadratic differential $\psi$ on it. Let $\pi: \tilde{M} \rightarrow M$ be the canonical double covering such that $\pi^{*} \psi=\omega^{2}$; by hypothesis, $\tilde{M}$ is connected. Let $2 n$ be the number of singularities of $\psi$ of odd degree; the double covering $\pi: \tilde{M} \rightarrow M$ has the ramification points exactly over these points. By the Riemann-Hurwitz formula the genus of the covering surface $\tilde{M}$ equals $2 g+n-1$. Hence

$$
\operatorname{dim} H_{1}\left(\tilde{M} ; \mathbb{Z}_{2}\right)=4 g+2 n-2 .
$$

We start the proof with a construction of a special basis of cycles on $\tilde{M}$ :

$$
a_{1}^{+}, b_{1}^{+}, a_{1}^{-}, b_{1}^{-}, \ldots, a_{g}^{+}, b_{g}^{+}, a_{g}^{-}, b_{g}^{-}, \tilde{c}_{1}, \ldots, \tilde{c}_{n-2}
$$


The basis is partially orthogonalized: the cycles $a_{i}^{ \pm}, b_{i}^{ \pm}$are obtained by lifting to $\tilde{M}$ closed paths on $M$ representing a symplectic basis in $H_{1}\left(M ; \mathbb{Z}_{2}\right)$. Thus $a_{i}^{+} \circ b_{i}^{+}=a_{i}^{-} \circ b_{i}^{-}=1$, and the other intersection indices for these cycles are zero. The cycles $\tilde{c}_{j}$ correspond to the "cuts" joining the consecutive pairs $P_{j}, P_{j+1}$ of ramification points of the covering; these cycles are completely analogous to the cycles from a standard Riemann-Hurwitz basis on a hyperelliptic surface.

The basis is constructed in such way that

$$
\operatorname{ind}_{a_{i}^{+}} \omega=\operatorname{ind}_{a_{i}^{-}} \omega \quad \operatorname{ind}_{b_{i}^{+}} \omega=\operatorname{ind}_{b_{i}^{-}} \omega,
$$

and $\operatorname{ind}_{\tilde{c}_{j}} \omega$ is easily expressed in terms of degrees $k_{j}$ and $k_{j+1}$ of the corresponding zeros $P_{j}, P_{j+1}$ of $\psi$. Thus:

$$
\begin{gathered}
\Phi(\omega)=\sum_{i=1}^{2 g}\left(\operatorname{ind}_{a_{i}^{+}}(\omega)+1\right)\left(\operatorname{ind}_{b_{i}^{+}}(\omega)+1\right)+\sum_{i=1}^{2 g}\left(\operatorname{ind}_{a_{i}^{-}}(\omega)+1\right)\left(\operatorname{ind}_{b_{i}^{-}}(\omega)+1\right)+ \\
+ \text { impact of the cycles } \tilde{c}_{j} \bmod 2= \\
=\text { impact of the cycles } \tilde{c}_{j} \bmod 2
\end{gathered}
$$

The remaining part of the theorem is an exercise in linear algebra and in arithmetic. We present now the complete proof.

We first treat the special case when $\psi$ has no singularities of odd degree at all. Note that in this case the covering $\pi: \tilde{M} \rightarrow M$ is a regular double covering. In particular, the monodromy of the covering along a closed path depends only on the homology class of the path. A closed path $\gamma$ on $M$ lifts to a closed path $\tilde{\gamma}$ on $\tilde{M}$ if and only if the corresponding cycle $[\gamma]$ belongs to $\operatorname{Ker}(m)$, where $m: H_{1}\left(M ; \mathbb{Z}_{2}\right) \rightarrow \mathbb{Z}_{2}$ is the monodromy homomorphism.

In the case when $\psi$ has no singularities of odd degree the basis of cycles on $\tilde{M}$ which we are going to construct has the form

$$
a_{1}^{+}, b_{1}^{+}, a_{1}^{-}, b_{1}^{-}, \ldots, a_{g-1}^{+}, b_{g-1}^{+}, a_{g-1}^{-}, b_{g-1}^{-}, a_{g}^{+}, \tilde{b}_{g},
$$

where there are only two cycles $a_{g}^{+}, \tilde{b}_{g}$ corresponding to index $g$. We obtain this basis of cycles on the covering surface $\tilde{M}$ using a special basis of cycles on the underlying surface $M$.

Let $a_{1}, b_{1}, \ldots, a_{g}, b_{g}$ be a canonical basis of $H_{1}\left(M, \mathbb{Z}_{2}\right)$, where $a_{i} \circ b_{i}=1$ for $i=1, \ldots, g$, and all the other intersection indices are trivial. Since the quadratic differential $\psi$ is not a global square of an Abelian differential, there exists a cycle $c \in H_{1}\left(M, \mathbb{Z}_{2}\right)$ with nontrivial monodromy. Without loss of generality we may assume that this cycle is $b_{g}$, that is $m\left(b_{g}\right)=1$. 
Let us perform now a change of the basis in $H_{1}\left(M, \mathbb{Z}_{2}\right)$ to obtain a canonical basis in $H_{1}\left(M ; \mathbb{Z}_{2}\right)$ with the additional property:

$$
\operatorname{ind}_{a_{i}} \psi=\operatorname{ind}_{b_{i}} \psi=0 \text { for } i=1, \ldots, g-1 \quad \text { and } \quad \operatorname{ind}_{a_{g}} \psi=0, \operatorname{ind}_{b_{g}} \psi=1
$$

We replace those $a_{i}, i<g$, and those $b_{j}, j<g$, that have nontrivial monodromy, by $a_{i}+b_{g}$ and $b_{j}+b_{g}$ correspondingly. Now we have $m\left(a_{i}\right)=m\left(b_{i}\right)=$ 0 for $i=1, \ldots, g-1$. It remains to adjust $a_{g}$ to get a canonical basis with desired properties.

We now use this basis on the underlying surface $M$ to construct a basis of cycles on the covering surface $\tilde{M}$. Since $m\left(a_{i}\right)=m\left(b_{i}\right)=0$ for $i=1, \ldots, g-1$, a closed path on $M$ representing any cycle from this collection can be lifted to a closed path on the covering surface $\tilde{M}$ in two different ways; the two corresponding lifts are disjoint. In this way we obtain the representatives of the cycles $a_{1}^{+}, b_{1}^{+}, a_{1}^{-}, b_{1}^{-}, \ldots, a_{g-1}^{+}, b_{g-1}^{+}$on $\tilde{M}$. We may assign the superscript indices \pm in such a way that $a_{i}^{+} \circ b_{i}^{+}=a_{i}^{-} \circ b_{i}^{-}=1$ and all the other intersection indices are zero. We now take a closed path on $M$ representing the cycle $a_{g}$ and we choose one of the two lifts of this path. The resulting closed path on $\tilde{M}$ gives us a cycle $a_{g}^{+} \in H_{1}\left(\tilde{M}, \mathbb{Z}_{2}\right)$. Since $m\left(b_{g}\right)=1$ we need to take a double lift of a representative of this cycle to get a closed path on $\tilde{M}$; it gives us $\tilde{b}_{g}$. We get a collection of $4 g-2$ cycles in $H_{1}\left(\tilde{M}, \mathbb{Z}_{2}\right)$. By construction the matrix of the intersection form for this collection has the canonical form; in particular, it is non-degenerate. Since the number of cycles equals the dimension of the homology group $H_{1}\left(\tilde{M} ; \mathbb{Z}_{2}\right)$, see equation (2), we get a canonical basis of cycles on the covering surface $\tilde{M}$.

Let us compute the parity of the spin structure of the Abelian differential $\omega$ on $\tilde{M}$ using this basis. By construction the basis is canonical, and equations (3) are valid for any $i=1, \ldots, g-1$. Thus:

$$
\begin{gathered}
\Phi(\omega)=\sum_{i=1}^{g-1}\left(\operatorname{ind}_{a_{i}^{+}} \omega+1\right)\left(\operatorname{ind}_{b_{i}^{+}} \omega+1\right)+\sum_{i=1}^{g-1}\left(\operatorname{ind}_{a_{i}^{-}} \omega+1\right)\left(\operatorname{ind}_{b_{i}^{-}} \omega+1\right)+ \\
+\left(\operatorname{ind}_{a_{g}^{+}}(\omega)+1\right)\left(\operatorname{ind}_{\tilde{b}_{g}}(\omega)+1\right) \bmod 2= \\
=\left(\operatorname{ind}_{a_{g}^{+}}(\omega)+1\right)\left(\operatorname{ind}_{\tilde{b}_{g}}(\omega)+1\right) \bmod 2
\end{gathered}
$$

By assumption $m\left(b_{g}\right)=1$. This means that the holonomy of the flat structure corresponding to $\psi$ along a smooth path on $M$ representing $b_{g}$ is an odd multiple of the angle $\pi$. Hence the holonomy of the flat structure corresponding to $\omega$ along a smooth path on $\tilde{M}$ which is a double cover of the corresponding path on $M$ is an odd multiple of the angle $2 \pi$. This means that $\operatorname{ind}_{\tilde{b}_{g}} \omega=$ 
$1 \bmod 2$, which implies $\Phi(\omega)=0$. We proved the Theorem in a special case when $\psi$ does not have poles no zeroes of odd degree. Moreover, we showed that the parity of the spin structure is always even in this special case.

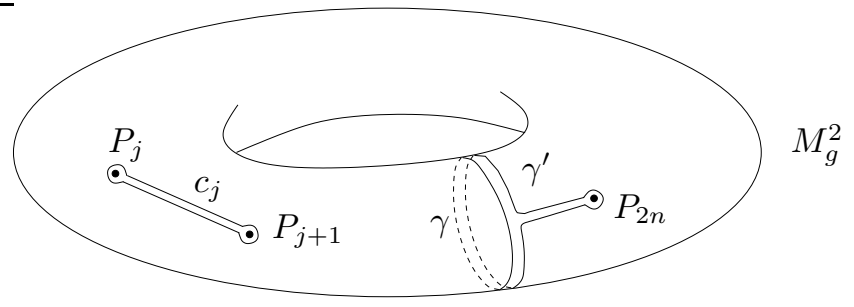

Figure 4: The points $P_{j}, P_{j+1}$, and $P_{2 n}$ of the underlying surface $M$ correspond to singularities of $\psi$ of odd degree. The paths $\gamma$ and $\gamma^{\prime}$ are homologous in $H_{1}\left(M ; \mathbb{Z}_{2}\right)$, but holonomy along $\gamma$ and along $\gamma^{\prime}$ is different. Holonomy along the path $c_{j}$ is obviously trivial.

Suppose now that $\psi$ has singularities of odd degree. Note that the number of such singularities is always even, we denote it by $2 n$. Now the covering $\pi: \tilde{M} \rightarrow M$ is ramified, and the holonomy homeomorphism is well-defined only for the punctured surface $M \backslash$ \{ramification points\}. Adding to a path $\gamma$ an appendix which goes to the ramification point, turns around it and then comes back, we get a new path representing the same homology class in $H_{1}\left(M ; \mathbb{Z}_{2}\right)$ but having different holonomy, see Figure 4. In particular, now we can choose a collection of smooth closed paths with trivial holonomy on the underlying surface $M$ representing a canonical basis of cycles $a_{i}, b_{i}$ in $H_{1}\left(M ; \mathbb{Z}_{2}\right)$. We denote these paths by the same symbols $a_{i}, b_{i}$ as the corresponding cycles.

Using this collection of paths on the underlying surface $M$ we construct a special basis of cycles

$$
a_{1}^{+}, b_{1}^{+}, a_{1}^{-}, b_{1}^{-}, \ldots, a_{g}^{+}, b_{g}^{+}, a_{g}^{-}, b_{g}^{-}, c_{1}, \ldots, c_{n-2}
$$

on the covering surface $\tilde{M}$. Here the cycles $a_{i}^{ \pm}, b_{i}^{ \pm}$correspond to the lifts of the paths $a_{i}, b_{i}$. We assign the superscript indices \pm to these lifts in such way that $a_{i}^{+} \circ b_{i}^{+}=a_{i}^{-} \circ b_{i}^{-}=1$.

The cycles $c_{j}$ are analogous to the ones from a standard Riemann-Hurwitz basis on a hyperelliptic surface. They are constructed as follows. For any $j=1, \ldots, 2 n-2$ consider a path on the underlying surface $M$ joining $P_{j}$ and $P_{j+1}$. We may choose this path in such way that it does not intersect any of the paths $a_{i}, b_{i}$, and that the corresponding "broken line" $P_{1}, P_{2}, \ldots, P_{2 g-1}$ does not have self-intersections. Deform these paths as on the Figure 4 to obtain 
closed loops $c_{j}$ with trivial monodromy. Lifting $c_{j}$ to a closed path $\tilde{c}_{j}$ on $\tilde{M}$ (in any of two possible ways) we get the desired cycles in $H_{1}\left(\tilde{M} ; \mathbb{Z}_{2}\right)$.

The subspace spanned by the cycles $a_{i}^{+}, a_{i}^{-}, b_{i}^{+}, b_{i}^{-}$, where $i=1, \ldots, g$, is orthogonal to the subspace spanned by the cycles $c_{j}$, where $j=1, \ldots, 2 n-2$. In the same way of the standard Riemann-Hurwitz basis on a hyperelliptic surface, we also have $\tilde{c}_{j} \circ \tilde{c}_{j+1}=1$ for $j=1, \ldots, 2 n-3$, and $\tilde{c}_{j_{1}} \circ \tilde{c}_{j_{2}}=0$ if $\left|j_{2}-j_{1}\right|>1$. Thus the matrix of the intersection form for this collection of cycles is non-degenerate. According to equation (2) the number $4 g+2 n-2$ of cycles equals the dimension of the homology group $H_{1}\left(\tilde{M} ; \mathbb{Z}_{2}\right)$. Hence we get a basis of cycles on the covering surface $\tilde{M}$.

Let us compute the parity of the spin structure of the Abelian differential $\omega$ on $\tilde{M}$ using this basis. The subspace $V_{1}$ spanned by the cycles $a_{i}^{+}, a_{i}^{-}, b_{i}^{+}, b_{i}^{-}$, where $i=1, \ldots, g$, is orthogonal to the subspace spanned by the cycles $c_{j}$, where $j=1, \ldots, 2 n-2$. By construction the cycles $a_{i}^{+}, b_{i}^{+}, a_{i}^{-}, b_{i}^{-}$give us a canonical basis of the subspace $V_{1}$, and for any $i=1, \ldots, g$ we have relations (3). Hence

$$
\sum_{i=1}^{2 g}\left(\operatorname{ind}_{a_{i}^{+}} \omega+1\right)\left(\operatorname{ind}_{b_{i}^{+}} \omega+1\right)=\sum_{i=1}^{2 g}\left(\operatorname{ind}_{a_{i}^{-}} \omega+1\right)\left(\operatorname{ind}_{b_{i}^{-}} \omega+1\right)
$$

which justifies equation (4). We have shown that the parity of the spin structure depends only of computation of index of cycles $\tilde{c}_{j}$. Thus this number depends only of degree of the singularities of $\omega$ that is the number $k_{i}$. This completes the proof of Theorem 4.1 .

\subsection{Explicit formula}

In this section, we give an explicit formula for the parity of the spin structure.

Theorem 4.2 Let $\psi$ be a meromorphic quadratic differential on a Riemann surface $M$ with singularity pattern $\mathcal{Q}\left(k_{1}, \ldots, k_{l}\right)$. Let $n_{+1}$ be the number of zeros of $\psi$ of degrees $k_{i}=1 \bmod 4$, let $n_{-1}$ be the number of zeros of $\psi$ of degrees $k_{j}=3 \bmod 4$, and suppose that the degrees of all the remaining zeros satisfy $k_{r}=0 \bmod 4$. Then the parity of the spin structure defined by $\psi$ is given by

$$
\Phi(\psi)=\left[\frac{\left|n_{+1}-n_{-1}\right|}{4}\right] \bmod 2
$$

where square brackets denote the integer part. 
Proof of the Theorem 4.2 Let $M$ be a Riemann surface endowed with a quadratic differential $\psi$. Let $\pi: \tilde{M} \rightarrow M$ be canonical double covering such that $\pi^{*} \psi=\omega^{2}$. We want to compute $\Phi(\psi)=\Phi(\omega)$. If $\psi$ has no singularities of odd degree at all (that is neither zeroes of odd degree, nor poles) then we already showed that $\Phi=0$ which proves the Theorem 4.2 in a special case. Suppose now that $\psi$ has singularities of odd degree. We use the notations in the proof of the Theorem 4.1. According to the equation (41), we have:

$$
\begin{gathered}
\Phi(\psi)=\sum_{i=1}^{2 g}\left(\operatorname{ind}_{a_{i}^{+}}(\omega)+1\right)\left(\operatorname{ind}_{b_{i}^{+}}(\omega)+1\right)+\sum_{i=1}^{2 g}\left(\operatorname{ind}_{a_{i}^{-}}(\omega)+1\right)\left(\operatorname{ind}_{b_{i}^{-}}(\omega)+1\right)+ \\
+ \text { impact of the cycles } \tilde{c}_{j} \bmod 2= \\
=\text { impact of the cycles } \tilde{c}_{j} \bmod 2
\end{gathered}
$$

If $\psi$ has only two singularities of odd degree, our basis does not have any cycles $\tilde{c}_{j}$ at all, so in this case the Theorem is proved. Suppose now that $\psi$ has more than two singularities of odd degree.

Let us compute now $\operatorname{ind}_{\tilde{c}_{j}} \omega$. We have to compute how the tangent vector to the corresponding path $\tilde{c}_{j}$ on $\tilde{M}$ turns in the flat structure defined by $\omega$, see Figure 5. The counterclockwise direction is chosen as a direction of the positive turn.

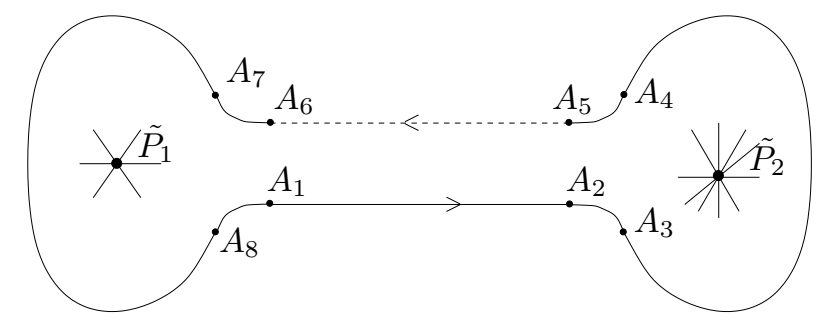

Figure 5: A path $\tilde{c}_{j}$ on the covering surface obtained by lifting the path $\tilde{c}_{j}$. Since $\tilde{P}_{j}, \tilde{P}_{j+1} \in \tilde{M}$ are ramification points, the segments $A_{1} A_{2}$ and $A_{6} A_{5}$ are located on "different sheets" of the covering $\tilde{M} \rightarrow M$, and are not near to one another.

We get some angle $\phi$ following the part $A_{1} A_{2}$ of the path which goes from one singularity to another. Then we make a turn by $-\pi / 2$ going along $A_{2} A_{3}$. Turning around the singularity (the path $\left.A_{3} A_{4}\right)$ we get the angle $\left(k_{j+1}+2\right) \pi$, which is followed by another turn by $-\pi / 2$ now along $A_{4} A_{5}$. The path $A_{5} A_{6}$ gives the turn by $-\phi$, which is followed by another $-\pi / 2$ along $A_{6} A_{7}$. Turning around singularity along $A_{7} A_{8}$ we get the angle $\left(k_{j}+2\right) \pi$, and the loop is completed by the path $A_{8} A_{1}$ giving one more turn by $-\pi / 2$. All together this 
gives $\left(k_{j}+k_{j+1}+2\right) \pi=\left(\left(k_{j}+k_{j+1}\right) / 2+1\right) \cdot 2 \pi$. So we obtain $\operatorname{ind}_{\tilde{c}_{j}}(\omega)=$ $\left(k_{j}+k_{j+1}\right) / 2+1$. Thus the value of the $\mathbb{Z}_{2}$-quadratic form $\Omega_{\omega}$ on the cycle $\tilde{c}_{j}$ is equal to

$$
\Omega_{\omega}\left(\tilde{c}_{j}\right)=\operatorname{ind}_{\tilde{c}_{j}}(\omega)+1=\frac{k_{j}+k_{j+1}}{2} \bmod 2
$$

Applying the Gram-Schmidt algorithm to the family $\tilde{c}_{j}$ we get the following symplectic basis $\alpha_{j}, \beta_{j}, j=1, \ldots, n-1$ in the subspace $V_{2} \subset H_{1}\left(\tilde{M} ; \mathbb{Z}_{2}\right)$ spanned by the cycles $\tilde{c}_{j}$ :

$$
\begin{array}{ccc}
\alpha_{1}=\tilde{c}_{1} & \beta_{1}=\tilde{c}_{2} \\
\alpha_{2}=\tilde{c}_{3}+\alpha_{1} & \beta_{2}=\tilde{c}_{4} \\
\alpha_{3}=\tilde{c}_{5}+\alpha_{2} & \beta_{3}=\tilde{c}_{6} \\
\vdots & \vdots \\
\alpha_{n-1}=\tilde{c}_{2 n-3}+\alpha_{n-2} & \beta_{n-1}=\tilde{c}_{2 n-2}
\end{array}
$$

Then

$$
\Phi(\psi):=\Phi(\omega)=\sum_{j=1}^{n-1} \Omega_{\omega}\left(\alpha_{j}\right) \cdot \Omega_{\omega}\left(\beta_{j}\right) \bmod 2 .
$$

We use formula (11) to evaluate the $\mathbb{Z}_{2}$-quadratic form $\Omega_{\omega}$ for this new bases:

$$
\Omega_{\omega}\left(\alpha_{j}\right)=\Omega_{\omega}\left(\tilde{c}_{1}+\tilde{c}_{3}+\cdots+\tilde{c}_{2 j-1}\right)=\Omega_{\omega}\left(\tilde{c}_{1}\right)+\Omega_{\omega}\left(\tilde{c}_{3}\right)+\cdots+\Omega_{\omega}\left(\tilde{c}_{2 j-1}\right),
$$

where we take into consideration that $\tilde{c}_{2 j_{1}-1} \circ \tilde{c}_{2 j_{2}-1}=0$ for any $j_{1}, j_{2}$. Since $\Omega_{\omega}\left(\beta_{i}\right)=\Omega_{\omega}\left(\tilde{c}_{2 i}\right)$ we finally obtain:

$$
\Phi(\psi)= \begin{cases}\frac{1}{4} \sum_{j=1}^{n-1}\left(k_{1}+k_{2}+\cdots+k_{2 j}\right)\left(k_{2 j}+k_{2 j+1}\right) \bmod 2 & \text { if } n \geq 2 \\ 0 & \text { if } n=0,1\end{cases}
$$

To complete the proof of the Theorem we need to apply several elementary arithmetic arguments. Assume that $n>1$. First note that all the integers $k_{j}$ are odd. Hence inside any pair of parentheses we have an even number which implies that we may replace the numbers $k_{j}$ in (5) by their residues modulo 4 without affecting the total sum modulo 2 .

We may enumerate the singularities of odd degrees in such way that the first $2 m$ ones $P_{1}, \ldots, P_{2 m}$ the residues $k_{j} \bmod 4$ alternate, and for the remaining $2(n-m-1)$ ones $P_{2 m+1}, \ldots, P_{2 n-2}$ the residues $k_{i} \bmod 4$ are the same. Then, 
for any $j \leq m$ we get $k_{1}+\cdots+k_{2 j}=0 \bmod 4$, and hence:

$$
\begin{aligned}
\Phi(\psi)=\frac{1}{4} \sum_{j=1}^{n-1}\left(k_{1}+k_{2}+\cdots\right. & \left.+k_{2 j}\right)\left(k_{2 j}+k_{2 j+1}\right) \bmod 2= \\
= & \frac{1}{4} \sum_{j=m+1}^{n-1}\left(k_{2 m+1}+\cdots+k_{2 j}\right)\left(k_{2 j}+k_{2 j+1}\right) \bmod 2
\end{aligned}
$$

Thus it is sufficient to check the formula in the statement of the Theorem only for two cases: when all zeros have degree 1, and when all zeros have degree 3 . Taking into consideration that for the total sum we have

$$
k_{1}+\cdots+k_{2 n-2}=0 \bmod 4
$$

we easily obtain the desired relation.

\subsection{Ergodic components of the Teichmüller geodesic flow}

Recall that the two invariants which classify connected components of the strata of $\mathcal{H}_{g}$ are the hyperellipticity and the spin structure. In the previous section, we have shown that the parity of the spin structure is constant on each stratum of the moduli space of meromorphic quadratic differential $\mathcal{Q}_{g}$. Moreover, we can calculate all hyperelliptic components of the moduli space of quadratic differentials. In [13, we proved that the strata $\mathcal{Q}(12)$ and $\mathcal{Q}(-1,9)$ do not possess an hyperelliptic component. However, it was proved by A Zorich by

a direct computation of the corresponding extended Rauzy classes that each of these two strata has exactly two distinct connected components. Using formula of Theorem 4.2, we get that the spin structure is even on these two strata and so, it does not distinguish the component; which gives a negative response to a question of Kontsevich and Zorich.

\section{A Some applications}

\section{A.1 Billiard Flow}

Let $P$ denote a polygon in $\mathbb{R}^{2}$. The billiard flow is given by the motion of a point with the usual optical reflection rule on the boundary $\partial P$ of $P$. A line element of this flow (the geodesic flow) is given by a point $x \in P$ and a direction $\theta \in \mathbb{R} / 2 \pi \mathbb{Z}$ on the unit tangent bundle of $P$. Orbits of this flow fail to have 
continuations when they hit the boundary of $P$. We would like trajectories to reflect off the boundary. If $e_{i}$ is an edge of $P$ and $\rho_{i}: S^{1} \rightarrow S^{1}$ represent the reflexion through $e_{i}$ then we identify $(p, v)$ with $\left(p, \rho_{i}(v)\right)$ for each $p \in e_{i}$.

Let $\Gamma \subset O(2)$ be the group generated by the reflections in sides. We are interested only by the case when $\Gamma$ is finite; in such case the polygon is called rational. An equivalent condition, when $P$ is simply connected, is that all angles are rational multiple of $\pi$. For a rational billiard, a classical construction (see [17] for a nice review of rational billiards) gives rise to a flat surface $\tilde{M}_{g}^{2}$ of genus $g$ i.e. a Riemann surface endowed with an Abelian differential. We recall here the construction.

Let $P \subset \mathbb{C}$ and $\Gamma$ as above. Take $|\Gamma|$ disjoint copies of $P$, each rotated by an element of $\Gamma$. For each copy $P_{c}$ of $P$ and each reflection $r \in \Gamma$, glue each edge $E_{c}$ of $P_{c}$ to the edge $r\left(E_{c}\right)$ of $r\left(P_{c}\right)$. When the group $\Gamma$ is finite, the result is a compact Riemann surface $\tilde{M}$

$$
\tilde{M}=\left(\bigsqcup_{\gamma \in \Gamma} \gamma(P)\right) / \sim
$$

where $\sim$ is the relation above. The form $d z$ on each copy $\gamma(P), \gamma \in \Gamma$, induces a holomorphic $1-$ form $\tilde{\omega}$ on $\tilde{M}$. It is easy to check that the singularities of $\tilde{\omega}$ are located at the vertices of the copies of $P$. Moreover, the billiard flow descends to the geodesic flow on the flat surface $(\tilde{M}, \tilde{\omega})$. When the billiard $P$ has symmetries, we can construct the smaller surface denoted by $\left(\tilde{M}_{1}, \tilde{\omega}_{1}\right)$, obtained by identifying $\gamma_{1}(P)$ and $\gamma_{2}(P)$ if they differ by a translation. We can deduce $(\tilde{M}, \tilde{\omega})$ from $\left(\tilde{M}_{1}, \tilde{\omega}_{1}\right)$ by a finite covering

$$
(\tilde{M}, \tilde{\omega}) \rightarrow\left(\tilde{M}_{1}, \tilde{\omega}_{1}\right) .
$$

The genus of $\tilde{M}$ is given in terms of the angles of $P$.

Thus a rational billiard defines a point $[\tilde{M}, \tilde{\omega}]$ in some stratum of moduli space $\mathcal{H}_{g}$. It is easy to compute the singularity pattern of $(\tilde{M}, \tilde{\omega})$ knowing the polygon $P$ and hence identify the stratum. It is, somehow, more complicated to identify the connected component of the stratum. The invariance of the spin-structure on each stratum of quadratic differential gives information about the connected components of the stratum which contains the surface $\tilde{M}$.

\section{A.2 Billiard table and Abelian differential}

For this section we refer to [17. Let $P$ be a rational polygonal billiard: that is a rational polygon with the billiard flow inside. Let $\left(m_{i} / n_{i}\right) \cdot \pi$ be the angles 
of $\pi$ with the convention that $\operatorname{gcd}\left(n_{i}, m_{i}\right)=1$. Let $N=\operatorname{lcm}\left(n_{i}\right)$ be the least common multiple of $n_{i}$. Denote by $k$ the number of sides of $P$. Let $\tilde{M}$ be the Riemann surface with the holomorphic 1 -form $\tilde{\omega}$ which arises from $P$. We can calculate the genus $\tilde{g}$ of $\tilde{M}$ as follows:

$$
\tilde{g}=1+\frac{N}{2}\left(k-2-\sum \frac{1}{n_{i}}\right)
$$

We can also calculate singularities of $\tilde{\omega}$. Each vertex of $P$ of angle $\left(m_{i} / n_{i}\right) \cdot \pi$, induces $N / n_{i}$ singularities of order $m_{i}-1$ of the form $\tilde{\omega}$. If $m_{i}=1$, the singularity is a fake zero (regular point of $\tilde{\omega}$ ) and we do not mark it.

\section{A.3 Billiard table and quadratic differential}

\section{A.3.1 Construction}

Here we present an alternative construction which associates a surface $M$ with a quadratic differential $\psi$ from a rational billiard $P$ (see [8]). The image of the holonomy map for the flat metric defined by $\psi$ on $M$ is contained in $\{ \pm \mathrm{Id}\}$. Let $\mathbb{P}^{1}$ be the double of $P$ : we consider two copies of $P$; one a top of the other and we identify the boundaries of these two copies gluing the corresponding edges. We obtain the topological sphere with a Euclidean metric. The segments coming from edges of the polygon are non-singular for this metric, so it has only isolated singularities coming from the vertices of the polygon. The singularities are conical singularities of angle rational multiple of $\pi$. We construct the standard covering of this surface to obtain a translation surface (not necessarily with nontrivial holonomy). The quadratic differential $\psi$ which we obtain is a square of an Abelian differential if and only if the $N=\operatorname{lcm}\left(n_{i}\right)$ is odd. Otherwise the surfaces $(\tilde{M}, \tilde{\omega})$ and $(M, \psi)$ are related by the standard double covering.

$$
(\tilde{M}, \tilde{\omega}) \rightarrow(M, \psi)
$$

When $N$ is odd, the two constructions coincide. We compute the singularity pattern of the surface $(M, \psi)$ as follow. Let $p_{i} / q_{i}$ denote the rational factor of $\pi / 2$ for angles of $P$ with $p_{i}$ and $q_{i}$ coprime. Let $Q$ be the $l c m$ of $q_{i}$. Then for each vertex, the quadratic differential $\psi$ on $M$ possesses $Q / q_{i}$ singularities of order $p_{i}-2$.

\section{A.3.2 Formula}

When the invariant surface $(\tilde{M}, \tilde{\omega})$ constructed by a rational billiard belongs to a non-connected stratum, we can apply Theorem 4.2 to identify the connected 
component which contain the point $[\tilde{M}, \tilde{\omega}]$. Let $P$ be a rational billiard. Let the angles of $P$ have the form $\left(m_{i} / n_{i}\right) \pi$ with $m_{i}=2 k_{i}+1$. This condition guarantees that the spin structure of the corresponding Abelian differential is well defined; that is all zeroes of $\omega$ are even. We enumerate the angles as follows: for $i=1, \ldots, r_{1}$, let $\left(m_{i} / n_{i}\right) \pi$ be the angles with $n_{i}$ even and $k_{i}$ even; for $i=r_{1}+1, \ldots, r_{1}+r_{2}$, let $\left(m_{i} / n_{i}\right) \pi$ be the angles with $n_{i}$ even and $k_{i}$ odd; finally for $i=1+r_{1}+r_{2}, \ldots, r+r_{1}+r_{2}$, let $\left(m_{i} / n_{i}\right) \pi$ be the angles with $n_{i}$ odd. We denote by $N$ the lcm of the $n_{i}$. Then we have the following

Corollary A.1 Let $P$ be a billiard table as above. Then the Abelian differential $\tilde{\omega}$ corresponding to $P$ has the following parity of the spin-structure:

$$
\Phi(\tilde{\omega})=\left[\frac{N}{4} \cdot\left|\sum_{i=1}^{r_{1}} \frac{1}{n_{i}}-\sum_{i=1+r_{1}}^{r_{1}+r_{2}} \frac{1}{n_{i}}\right|\right] \bmod 2
$$

Proof First we calculate angles with the form $\frac{p_{i}}{q_{i}} \cdot \frac{\pi}{2}$ with $p_{i}$ and $q_{i}$ coprimes. With considerations above, it is not difficult to see that we can take $\left(p_{i} ; q_{i}\right)=$ $\left(m_{i} ; \frac{n_{i}}{2}\right)$ for $i=1, \ldots, r_{1}+r_{2}$ and $\left(p_{i} ; q_{i}\right)=\left(2 \cdot m_{i} ; n_{i}\right)$ for $i=1+r_{1}+$ $r_{2}, \ldots, r+r_{1}+r_{2}$. The degrees of the singularities of the corresponding quadratic differential are given by the $p_{i}$. For $i=1, \ldots, r_{1}+r_{2}$, we obtain zeroes of degree $p_{i}-2=m_{i}-2=2 k_{i}-1$; for $i=1+r_{1}+r_{2}, \ldots, r+r_{1}+r_{2}$, we obtain zeroes of degree $p_{i}-2=2 \cdot\left(m_{i}-1\right)$. In the last case, all zeroes are even so it does not appear in the formula of Theorem 4.2. When $k_{i}$ is even, the corresponding $p_{i}$ is equal to -1 modulo 4 and $k_{i}$ is odd, the corresponding $p_{i}$ is equal to +1 modulo 4. Let us calculate the multiplicities of the zeroes. Let $Q$ denote the lcm of the $q_{i}$. Now, the formula of the corollary follows from Theorem 4.2 and the following fact

$$
Q=\frac{N}{2} ; \quad q_{i}=\frac{n_{i}}{2}
$$

Quite often the translation surface $(\tilde{M}, \tilde{\omega})$ corresponding to a rational billiard $P$ is hyperelliptic. Corollary A.1 allows one to determine the case when it is not, see $\mathbf{A . 3 . 3}$. Though the surfaces $(\tilde{M}, \tilde{\omega})$ arising from billiards form subsets of measure zero in corresponding strata, the properties of the ambient strata have strong influence on the dynamics of the billiard. In particular billiards inducing hyperelliptic and non-hyperelliptic surfaces have different behavior. 


\section{A.3.3 Hyperelliptic Components}

For moduli space of Abelian differentials, the parity of the spin structure of the hyperelliptic components is well defined. In 12, Kontsevich and Zorich give a formula for calculation of these numbers for any hyperelliptic component. This is given by the following Proposition

Proposition (Kontsevich, Zorich) The parity of the spin structure for a translation surface in a hyperelliptic component with one singularity and for a translation surface in a hyperelliptic component with two singularities is given, respectively, by

$$
\Phi\left(\mathcal{H}(2 g-2)^{h y p}\right)=\left[\frac{g+1}{2}\right] \bmod 2
$$

and

$$
\Phi\left(\mathcal{H}(g-1, g-1)^{h y p}\right)=\frac{g+1}{2} \bmod 2, \text { for } g \text { odd }
$$

Example Consider the polygon given by the triangle $(11 \pi / 14 ; \pi / 7 ; \pi / 14)$. We consider the billiard flow inside this polygon. The number $N$ appearing in the classical construction $A .2$. is $N=14$. In particular it is even. The corresponding Abelian differential $(\tilde{M}, \tilde{\omega})$ has 2 fake zeroes and a zero of order 10 . Thus it determines a point in the stratum $\mathcal{H}(10)$. The surface $\tilde{M}$ has genus 6 . We can calculate the singularity pattern of the corresponding quadratic differential. The angles are given by the rational factor of $\pi$ : $\left(\frac{2 \cdot 5+1}{14} ; \frac{2 \cdot 0+1}{14} ; \frac{1}{7}\right)$. Thus by Corollary A.1, the parity of the spin structure of $(\tilde{M}, \tilde{\omega})$ is even.

According to formula 6 the parity of the spin structure of the hyperelliptic component of the stratum $\mathcal{H}(10)$ is odd and so the surface $\tilde{M}$ is not hyperelliptic, and $[\tilde{M}, \tilde{\omega}] \in \mathcal{H}^{\text {odd }}(10)$. 


\section{References}

[1] C Arf, Untersuchungen über quadratische Formen in Körpern der Charakteristik 2, (in German) J. Reine Angew. Math. 183 (1941) 148-167

[2] M Atiyah, Riemann surfaces and spin structures, Ann. Scient. Ec. Norm. Sup. 4 (1971) 47-62

[3] A Douady, J Hubbard, On the density of Strebel differentials, Invent. Math. 30 (1975) 175-179

[4] A Eskin, H Masur, A Zorich, Moduli spaces of Abelian differentials: the principal boundary, counting problems and the Siegel-Veech constants, Publ. Math. Inst. Hautes Études Sci. 97 (2003) 61-179

[5] A Eskin, A Okounkov, Asymptotics of number of branched coverings of a torus and volumes of moduli spaces of holomorphic differentials, Invent. Math. 145 (2001) 59-104

[6] A Fathi, F Laudenbach, V Poenaru, Travaux de Thurston sur les surfaces, Astérisque 66-67 (1979)

[7] H Karkas, I Kra, Riemann surfaces, Second edition, Graduate Texts in Mathematics 71 Springer-Verlag, New York (1992)

[8] R H Fox, R B Kershner, Geodesics on a rational polyhedron, Duke Math. J. 2 (1936) $147-150$

[9] J Hubbard, H Masur, Quadratic differentials and foliations, Acta Math. 142 (1979) 221-274

[10] D Johnson, Spin structures and quadratic forms on surfaces, J. London Math. Soc. 22 (1980) 365-373

[11] M Kontsevich, Lyapunov exponents and Hodge theory, from: "The mathematical beauty of physics (Saclay, 1996) in Honor of C Itzykson", Adv. Ser. Math. Phys. 24, World Sci. Publishing, River Edge, NJ (1997) 318-332

[12] M Kontsevich, A Zorich, Connected components of the moduli spaces of Abelian differentials with prescribed singularities, Invent. Math. 153 (2003) 631678

[13] E Lanneau, Hyperelliptic connected components of the moduli spaces of quadratic differentials, Comment. Math. Helvetici, to appear (2004)

[14] E Lanneau, Classification of connected components of the strata of the moduli spaces of quadratic differentials with prescribed singularities, Thèse de Doctorat, Université de Rennes 1 (2003)

[15] H Masur, Interval exchange transformations and measured foliations, Ann. of Math. 115 (1982) 169-200

[16] H Masur, J Smillie, Quadratic differentials with prescribed singularities and pseudo-Anosov diffeomorphisms, Comment. Math. Helvetici 68 (1993) 289-307 
[17] H Masur, S Tabachnikov, Flat structures and rational billiards, from: "Handbook of dynamical systems Vol. 1A", North-Holland Amsterdam (2002) 10151089

[18] J Milnor, Remarks concerning spin manifolds, from: "Differential and combinatorial topology (Symposium in honor of Marston Morse)", Princeton (1965) $55-62$

[19] D Mumford, Theta-characteristics of an algebraic curve, Ann. scient. Éc. Norm. Sup. 2 (1971) 181-191

[20] G Rauzy, Échanges d'intervalles et transformations induites, (French), Acta Arith. 34 (1979) 315-328

[21] K Strebel, Quadratic differentials, Springer-Verlag, (1984)

[22] W A Veech, Gauss measures for transformations on the space of interval exchange maps, Ann. of Math. 115 (1982) 201-242

[23] W A Veech, Moduli spaces of quadratic differentials, Journal d'Analyse Math. 55 (1990) 117-171 